\title{
A DIVULGAÇÃO DE VÍDEOS DE ANATOMIA DO SISTEMA NERVOSO CENTRAL NO YOUTUBE
}

\section{Gustavo Vieira de Oliveira}

Doutorando em Ciência, Programa de pós-graduação em Produtos Bioativos e Biociência, Universidade Federal do Rio de Janeiro, Macaé (RJ), Brasil.

\section{Paula Alvarez Abreu}

Doutora em Neurociência. Laboratório de Modelagem molecular e Pesquisa em Ciências Farmacêuticas (LAMCIFAR), Universidade Federal do Rio de Janeiro, Macaé (RJ), Brasil.
RESUMO: O estudo visa avaliar os métodos de exposição de conteúdos de neuroanatomia utilizados nos vídeos compartilhados no YouTube, o impacto destes vídeos e a aceitação dos usuários. Trata-se de uma pesquisa quantitativa, com abordagem descritiva e analítica observacional, envolvendo a análise de vídeos de neuroanatomia divulgados em português no site do YouTube. A pesquisa foi realizada no Youtube utilizando as palavras-chave "telencéfalo", "diencéfalo" e "tronco encefálico". Os vídeos foram categorizados de acordo com o método de exposição do conteúdo de neuroanatomia como equipamentos multimídia (EM), peças plásticas (PPT), peças plastinadas (PPN), e peças cadavéricas formolizadas (PCF) para avaliação do número de visualizações e aceitação dos usuários (número de likes e dislikes). Foi observado que as PPT foram mais utilizadas nos vídeos de neuroanatomia. Porém, os vídeos que utilizaram EM tiveram mais visualizações diárias e maior aceitação pelos usuários do Youtube. Os resultados do presente estudo sugerem que os vídeos de neuroanatomia utilizando EM podem contribuir de forma complementar para o ensino-aprendizado destes conteúdos.

PALAVRAS-CHAVE: Educação; Neuroanatomia; Anatomia.

\section{DISSEMINATION OF VIDEOS ON THE ANATOMY OF THE CENTRAL NERVOUS SYSTEM BY YOUTUBE}

ABSTRACT: The aim of this work is to access the exposure methods of neuroanatomy used in YouTube-shared videos, their impact and acceptance by users, by a quantitative, descriptive and analytic research. Research was undertaken in YouTube with keywords "telencephalon", "diencephalon" and "brainstem". Videos were categorized according to the exposure method of the neuroanatomic contents, such as multimedia equipments (ME), plastic parts (PPT), plastinated parts (PPN), and cadaver pieces in formaldehyde (PCF), to evaluate the number of visualizations and acceptance by the users (number of likes and dislikes). The exposure method based on PPT was the most used one in neuroanatomy videos. However, ME videos had more daily visualizations and greater acceptance by the Youtube users. Results suggest that neuroanatomy videos with ME may contribute in a complementary manner towards the teaching-learning of these contents.

KEY WORDS: Education; Neuroanatomy; Anatomy. 


\section{INTRODUÇÃO}

A essência do ensino da anatomia humana tem mudado substancialmente nas últimas décadas. Por séculos, a anatomia foi ensinada por meio de muitas horas disponibilizadas à dissecção de cadáveres. No entanto, o avanço da tecnologia tem transformado a maneira como os estudantes aprendem estes conteúdos ${ }^{1}$.

A anatomia humana é uma disciplina que faz parte do currículo de diversos cursos de graduação, pós-graduação e profissionalizante na área da saúde. O conhecimento de anatomia é essencial para profissionais que atuam nas áreas clínicas, principalmente os cirurgiões e radiologistas $^{2}$. A neuroanatomia apresenta-se como um segmento da anatomia humana que envolve o estudo do sistema nervoso central e periférico. O entendimento do sistema nervoso central é essencial na medicina, especialmente para as especializações em neurologia, neurocirurgia e psiquiatria. Estas especializações requerem conhecimento avançado do encéfalo, o qual é dividido didaticamente em telencéfalo, diencéfalo e tronco encefálico ${ }^{3}$.

A educação médica moderna tem buscado a integração curricular, caracterizada pelo ensino simultâneo da ciência básica e clínica nos primeiros anos da graduaçãó . Nessa perspectiva, os estudantes são orientados a pesquisarem informações adicionais na internet, a qual é um recurso de fácil acesso, baixo custo e com muito conteúdo disponível'5.

A tecnologia dos computadores e a internet são componentes integrais do cotidiano dos estudantes das áreas biológicas e da saúde ${ }^{6-7}$. Sendo assim, diversos locais de rede como os de compartilhamento de vídeos (YouTube, Veoh), e de pesquisa (Google, Wikipédia) vêm sendo utilizados pelos alunos e permitem acesso rápido à informação ${ }^{8}$.

No YouTube, os usuários podem compartilhar, assistir e criar canais para divulgação de vídeos com temas específicos, e os proprietários dos canais podem emitir avisos aos assinantes acerca da divulgação de novos vídeos compartilhados. E neste contexto, o YouTube tem sido uma ferramenta útil no processo de ensino-aprendizagem de diversos conteúdos incluindo anatomia humana ${ }^{7}$.
Estudos têm mostrado que estudantes de medicina consideram os vídeos de anatomia humana disponíveis no YouTube uma ferramenta complementar útil para ampliar o conhecimento ${ }^{7,9}$. Azer ${ }^{10}$ avaliou os vídeos de anatomia de superfície, enquanto, Raikos e Waidyasekara ${ }^{11}$ avaliaram a quantidade e qualidade dos vídeos relacionados com a anatomia cardíaca e, além disso, demonstraram que o número de visualizações e a opinião (likes e dislikes) dos usuários do YouTube depende do método de exposição (e.g. peças plástica, cadavéricas, etc) do conteúdo de anatomia cardíaca.

Sendo assim, a avaliação dos métodos de exposição do conteúdo de anatomia utilizados nos vídeos compartilhados no YouTube e a opinião dos usuários se mostra importante para delinear estratégias mais efetivas para a produção de novos vídeos. Além disso, não há estudos avaliando os métodos de exposição do conteúdo de neuroanatomia e a opinião dos usuários do YouTube.

Portanto, considerando a importância do conhecimento da neuroanatomia para profissionais da saúde humana, a ampla divulgação e utilização de vídeos no YouTube para ensino-aprendizagem por diversos estudantes, o presente estudo tem como objetivo avaliar os métodos de exposição do conteúdo de neuroanatomia utilizados nos vídeos em português compartilhados no YouTube e o impacto dos vídeos medidos pela visualização e aceitação dos usuários.

\section{METODOLOGIA}

$\mathrm{O}$ presente estudo trata-se de uma pesquisa quantitativa, com abordagem descritiva e analítica observacional, envolvendo a avaliação de vídeos de neuroanatomia em português divulgados no YouTube. No período de 26 a 30 de maio de 2018 foi realizada uma busca por vídeos relacionados ao ensino de anatomia do sistema nervoso central no YouTube. Foram utilizadas as palavras-chave "telencéfalo", "diencéfalo" e "tronco encefálico" no campo de busca do YouTube (www.youtube. com). Foram excluídos os vídeos repetidos, sem áudio ou com fundo musical, com imagem ou áudio inadequado, que usaram mais de um método de exposição do conteúdo, com número de visualizações menor do que 100 
e duração menor do que 1 minuto, e aqueles em língua estrangeira. Somente os vídeos em língua portuguesa foram incluídos para análise uma vez que a proposta do presente estudo foi avaliar os vídeos de neuroanatomia divulgados no YouTube que poderiam contribuir para o processo de ensino-aprendizagem de estudantes brasileiros. Após elegibilidade, os vídeos foram categorizados de acordo com o método de exposição do conteúdo de neuroanatomia em peças plásticas (PPT), plastinadas (PPN); peças cadavéricas formolizadas (PCF) e equipamento multimídia (EM). Para avaliação do impacto (visualizações) e aceitação (likes ou dislikes) dos usuários foram realizados os seguintes cálculos: a) somatório do número de visualizações, likes e dislikes dos vídeos em cada categoria; b) o somatório do número de visualizações dos vídeos da categoria foi dividido pelo número de dias em que os vídeos estavam disponíveis no YouTube para obtenção do número de visualização/dia; c) o somatório do número de likes foi dividido pelo somatório do número de dislikes dos vídeos em cada categoria para obtenção do índice de aceitação dos vídeos (razão likes/dislikes).

\section{RESULTADOS}

Neste estudo foram avaliados os vídeos do YouTube relacionados ao ensino de anatomia do sistema nervoso central. As palavras-chave "telencéfalo", "diencéfalo" e "tronco encefálico" foram usadas separadamente e foram obtidos 5.150 vídeos de telencéfalo, 5.400 de diencéfalo e 7.480 de tronco encefálico. Após o uso dos critérios de exclusão foram elegíveis 45 vídeos de telencéfalo, 22 de diencéfalo e 39 de tronco encefálico; estes foram analisados separadamente nestes três grupos e foram categorizados de acordo com os métodos usados para exposição dos conteúdos, EM, PPT, PPN e PCF (Figura 1).

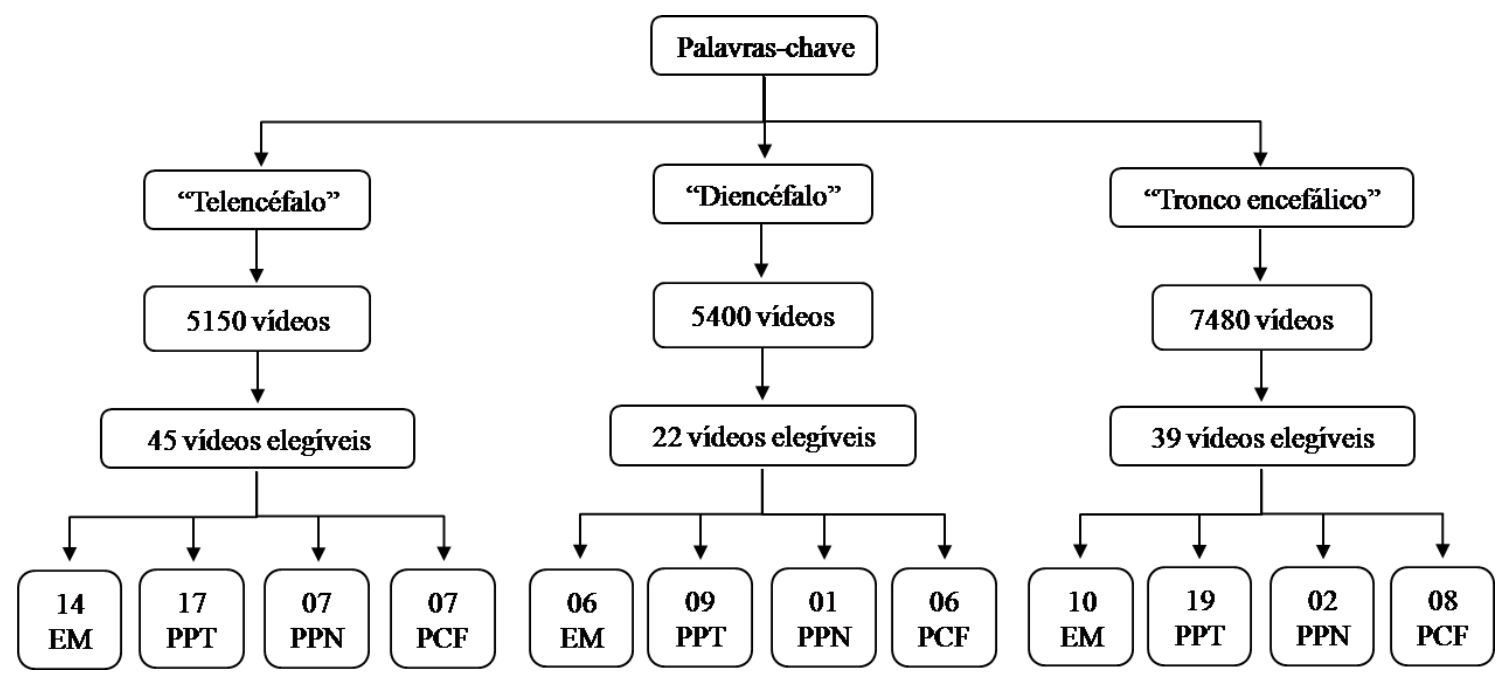

Figura 1. Fluxograma da busca dos vídeos de neuroanatomia no YouTube relacionando o número de vídeos encontrados, os elegíveis e os categorizados de acordo com o método de exposição do conteúdo.

Notas: $\mathrm{EM}=$ equipamento multimídia $;$ PPT $=$ peças plásticas; $\mathrm{PPN}=$ peças plastinadas $;$ PCF $=$ peças cadavéricas formolizadas

As análises dos vídeos relacionados ao telencéfalo mostraram que dos 45 vídeos elegíveis, 14 (31\%) utilizaram EM, 17 (37,8\%) PPT, 07 (15,6\%) PPN e sete (15,6\%) PCF. Os vídeos que utilizaram PPT tiveram maior número de visualizações (434.111) seguido de EM (355.036), PPN (189.643) e PCF (161.288). Com relação à aceitação dos vídeos, foi observado que aqueles que utilizaram EM tiveram maior número de likes (12.186) seguido de PPT (4.492), PPN (897) e PCF (351). Os vídeos que utilizaram
EM também tiveram maior número de dislikes (165) seguido de PPT (145), PPN (36) e PCF (20). Foi observado que vídeos que utilizaram EM apresentaram maior valor na razão likes/dislikes, seguido de PPT, PPN e PCF. Após cálculo da razão entre o número de visualizações pelo número de dias em que os vídeos estavam disponíveis, foi observado que os vídeos que utilizaram EM tiveram maiores valores de visualizações por dia, seguido de PPT, PPN e PCF (Tabela 1). 
Tabela 1. Análise da visualização e aceitação dos usuários pelos métodos de exposição (EM = equipamento multimídia; PPT $=$ peças plásticas PPN = peças plastinadas $;$ e PCF = peças cadavéricas formolizadas) do conteúdo telencéfalo utilizados na produção de vídeos de neuroanatomia divulgados no YoúTube

\begin{tabular}{cccccccc}
\hline & Vídeos $\mathrm{n}(\%)$ & Visualizações & No de dias & Visualizações/dia & like & dislike & $\begin{array}{c}\text { Razão like/ } \\
\text { dislike }\end{array}$ \\
\hline EM & $14(31 \%)$ & 355.036 & 13.428 & 26,4 & 12.186 & 165 & 74 \\
PPT & $17(37,8 \%)$ & 434.111 & 29.487 & 14,7 & 4.492 & 145 & 31 \\
PPN & $7(15,6 \%)$ & 189.643 & 12.921 & 14,6 & 897 & 36 & 25 \\
PCF & $7(15,6 \%)$ & 161.288 & 13.587 & 11,8 & 351 & 20 & 17 \\
\hline
\end{tabular}

As análises dos vídeos relacionados ao diencéfalo mostraram que dos 5.400 vídeos de diencéfalo encontrados, 22 (0,41\%) foram elegíveis. Dos 22 vídeos elegíveis, seis $(27,3 \%)$ utilizaram EM, nove (40,9\%) PPT, um (4,5\%) PPN e seis (27,3\%) PCF. Os vídeos que utilizaram EM tiveram maior número de visualizações (399.037) seguido de PPT (200.919), PCF (22.096) e PPN (361). Com relação à aceitação dos vídeos, também foi observado que os que utilizaram EM tiveram maior número de likes (13.534) seguido de PPT (1.908), PCF (121) e PPN (4). Os vídeos que utilizaram EM tiveram maior número de dislikes (66) também, seguido de PPT (59), PCF (4) e PPN (0). Foi observado que vídeos que utilizam EM apresentaram maior razão likes/dislikes, seguido de PPT e PCF. Não foi avaliada a razão likes/dislikes para PPN uma vez que não houve dislikes. Após cálculo da razão entre o número de visualizações pelo número de dias em que os vídeos estavam disponíveis, foi observado que os vídeos que utilizaram EM tiveram maiores valores de visualizações por dia, seguido de PPT, PCF e PPN (Tabela 2).

Tabela 2. Análise da visualização e aceitação dos usuários pelos métodos de exposição (EM = equipamento multimídia; PPT $=$ peças plásticas; PPN $=$ peças plastinadas; e PCF $=$ peças cadavéricas formolizadas) do conteúdo diencéfalo utilizados na produção de vídeos de neuroanatomia divulgados no YouTube

\begin{tabular}{cccccccc}
\hline & Vídeos n (\%) & Visualizações & No de dias & Visualizações/dia & like & dislike & $\begin{array}{c}\text { Razão like/ } \\
\text { dislike }\end{array}$ \\
\hline EM & $6(27,3 \%)$ & 399.037 & 5.229 & 76,3 & 13.534 & 66 & 205 \\
PPT & $9(40,9 \%)$ & 200.919 & 13.563 & 14,8 & 1.908 & 59 & 32 \\
PPN & $1(4,5 \%)$ & 361 & 861 & 0,4 & 4 & 0 & ND \\
PCF & $6(27,3 \%)$ & 22.096 & 8.328 & 2,6 & 121 & 04 & 30 \\
\hline
\end{tabular}

$\mathrm{ND}=$ Não determinado

As análises dos vídeos relacionados ao tronco encefálico mostraram que dos 7.480 vídeos de tronco encefálico encontrados, 39 (0,52\%) foram elegíveis. Dos 39 vídeos elegíveis, 10(25,6\%) utilizaram EM, 19 (48,7\%) PPT, dois $(5,1 \%)$ PPN e oito (20,5\%) PCF. Os vídeos que utilizaram PPT tiveram maior número de visualizações (179.066) seguido de EM (156.615), PCF (85.372) e PPN (11.793). Com relação à aceitação dos vídeos, também foi observado que os que utilizaram EM tiveram maior número de likes (3.308) seguido de PPT (1.281), PCF (370) e PPN (52). Os vídeos que utilizaram EM tiveram maior número de dislikes (84) seguido de PPT (31), PCF (17) e PPN (2). Foi observado que vídeos que utilizam PPT apresentaram maior razão like/dislike, seguido de EM, PPN e PCF. Após cálculo da razão entre o número de visualizações pelo número de dias em que os vídeos estavam disponíveis, foi observado que os vídeos que utilizaram EM tiveram maiores valores de visualizações por dia, seguido de PPT, PCF e PPN (Tabela 3). 
Além disso, considerando o total de vídeos elegíveis $(n=106)$, nos três segmentos do sistema nervoso central (telencéfalo, diencéfalo e tronco encefálico) analisado, foi observado que 45 vídeos (42,5\%) foram produzidos utilizando PPT, seguido de 30 vídeos (28,3\%) que usaram EM, 21 vídeos (19,8\%) que usaram PCF e dez vídeos $(9,4 \%)$ que usaram PPN. Para o total de visualizações $(n=2.195 .337)$, foi observado que os vídeos que utiliza- ram EM receberam 910.688 visualizações, correspondendo a 41,5\%, seguido de PPT com 814.096 visualizações (37,1\%), PCF com 268.756 visualizações $(12,2 \%)$ e PPN com 201.797 visualizações $(9,2 \%)$. Para o total de likes (n $=38.504$ ), foi observado que os vídeos que utilizaram EM receberam 29.028 likes que corresponde a 75,3\%, seguido de PPT com 7.681 likes (19,9\%), PPN com 953 likes $(2,4 \%)$ e PCF com 842 likes (2,1\%).

Tabela 3. Análise da visualização e aceitação dos usuários pelos métodos de exposição (EM = equipamento multimídia; PPT = peças plásticas; PPN = peças plastinadas; e PCF = peças cadavéricas formolizadas) do conteúdo tronco encefálico utilizados na produção de vídeos de neuroanatomia divulgados no YoúTube

\begin{tabular}{cccccccc}
\hline & $\begin{array}{c}\text { Vídeos } \\
\mathbf{n}(\%)\end{array}$ & Visualizações & No de dias & $\begin{array}{c}\text { Visualizações/ } \\
\text { dia }\end{array}$ & like & dislike & $\begin{array}{c}\text { Razão like/ } \\
\text { dislike }\end{array}$ \\
\hline EM & $10(25,6 \%)$ & 156.615 & 9.016 & 17,3 & 3.308 & 84 & 39 \\
PPT & $19(48,7 \%)$ & 179.066 & 35.914 & 4,9 & 1.281 & 31 & 41 \\
PPN & $2(5,1 \%)$ & 11.793 & 4.777 & 2,4 & 52 & 2 & 26 \\
PCF & $8(20,5 \%)$ & 85.372 & 13.868 & 6,1 & 370 & 17 & 22 \\
\hline
\end{tabular}

\section{DISCUSSÃO}

O presente estudo avaliou diferentes métodos de exposição do conteúdo de neuroanatomia em vídeos compartilhados no YouTube. Os vídeos analisados foram aqueles relacionados à anatomia do sistema nervoso central abrangendo os temas telencéfalo, diencéfalo e tronco encefálico, os quais foram usados como palavras-chave separadamente. Do total de vídeos em cada grupo observou-se que havia muitos vídeos repetidos e muitos foram excluídos por estarem em outro idioma, como espanhol. Foi possível observar neste estudo que os vídeos que utilizaram EM foram mais visualizados e aceitos (receberam mais likes).

Raikos e Waidyasekara ${ }^{11}$ investigaram os métodos de exposição do conteúdo de anatomia cardíaca (PPT, conferência, PPN, PCF, ilustrações e animações) nos vídeos compartilhados no YouTube. Os autores demonstraram que as PPT foram utilizadas em 54,4\% dos 294 vídeos analisados, seguido de peças PCF $(20,7 \%)$, conferência $(12,9 \%)$, ilustrações $(9,2 \%)$, animações $(1,7 \%)$ e PPN (1\%). Corroborando com estes achados, o presente estudo demonstrou que as PPT foram mais utilizadas na produção dos vídeos de neuroanatomia. A maior utilização de PPT pode estar relacionada à conveniência, utilidade $\mathrm{e}$ acessibilidade que estes recursos proporcionam na elaboração de material pedagógico. As estruturas anatômicas presentes nas PPT são mais simples e didáticas comparadas com peças cadavéricas e plastinadas. Além disso, a política de utilização de cadáveres tem limitado a aquisição e exposição deste material nos laboratórios de anatomia humana, o que pode acarretar no menor uso ${ }^{12}$. Tem sido demonstrado que o uso de materiais alternativos (não cadavéricos) pode ser uma estratégia pedagógica eficaz em neuroanatomia nos momentos de escassez de material cadavérico $^{13}$.

Raikos e Waidyasekara ${ }^{11}$ demonstraram que os vídeos de anatomia cardíaca com PPT foram mais visualizados. O presente estudo demonstrou que os vídeos de telencéfalo e tronco encefálico produzidos com PPT tiveram mais visualizações, enquanto os vídeos usando EM tiveram maior número de visualizações no segmento diencéfalo, bem como quando analisado o total de vídeos das três categorias. Quando considerado o valor relativo (razão entre o número de visualizações e os dias em que os vídeos estavam disponíveis no YouTube), os vídeos que utilizaram EM também foram identificados como que apresentaram maior visualização por dia. O 
YouTube recebe mais de 100 milhões de acessos por mês e, por isso, o número de visualizações de um determinado vídeo pode mudar diariamente. Os vídeos que estão disponíveis há mais dias apresentam maiores chances de serem visualizados. Este fato pode explicar os diferentes resultados obtidos quando se compara o número de visualizações e o número de visualizações por dias em que os vídeos estão disponíveis no YouTube. O uso da razão entre número de visualizações e o número de dias em que o vídeo estava disponível possibilita uma comparação mais adequada.

Os vídeos que utilizaram EM continham informações conceituais e aspectos clínicos acerca da neuroanatomia. Em contrapartida, os vídeos que utilizam PPT, PPN e PCF continham informações pontuais acerca das estruturas anatômicas presentes no material. Este fato sugere que os usuários do YouTube podem utilizar deste recurso audiovisual para complementar o conteúdo aplicado em sala de aula. Bergman et al. ${ }^{4}$ reportaram que, em algumas instituições, o número de estudante por professor tem dobrado e a média total de horas disponibilizadas para o ensino de anatomia humana tem sido reduzida em 50\%. Além disso, houve redução do uso do cadáver nas universidades devido as diversas inconveniências, como o forte cheiro de formol, alto custo e questões bioéticas ${ }^{14-15}$. Estes fatores podem dificultar o processo de ensino-aprendizagem, levando os estudantes a buscarem a internet para ampliar o conhecimento.

Os resultados demonstraram que os vídeos que utilizaram EM receberam mais likes, mas também foram os que receberam mais dislikes. Em contraste, Raikos e Waidyasekara ${ }^{11}$ demonstraram que os vídeos de anatomia cardíaca produzidos com PPT apresentaram maior número de likes e os que utilizaram ilustrações apresentaram maior número de dislikes. Apenas um vídeo foi observado utilizando ilustrações para expor o conteúdo de telencéfalo. No entanto, o vídeo foi excluído do nosso estudo por possuir menos do que 100 visualizações.

Um estudo demonstrou que estudantes de medicina consideram a neuroanatomia um dos segmentos mais complexos da anatomia humana ${ }^{3}$. Os estudantes reportaram que a dificuldade no entendimento da neuroanatomia tem relação com a falta de integração da ciência básica e clínica, complexidade do tema e ensino inade- quado 3 . Os vídeos que utilizam EM, geralmente abrangem aspectos conceituais da neuroanatomia, enquanto os vídeos que utilizam PPT, PPN e PCF abrangem aspectos morfológicos específicos (nomes das estruturas sem mencionar a função). Portanto, os nossos resultados sugerem que no segmento da anatomia humana do sistema nervoso central, o método de exposição do conteúdo nos vídeos parece influenciar a aceitação dos usuários do YouTube assim como parece ter ocorrido no estudo anterior com o sistema cardíaco ${ }^{11}$.

\section{CONCLUSÃO}

Foi demonstrado que $42,5 \%$ dos vídeos de neuroanatomia identificados em nosso estudo no YouTube foram produzidos utilizando PPT. No entanto, os vídeos que utilizaram EM tiveram maior número de visualizações (41,5\%) e também maior número de visualizações por dia nas três categorias (telencéfalo, diencéfalo e tronco encefálico), além de terem sido os mais aceitos pelos usuários (com $75,3 \%$ de likes). Estes resultados são importantes do ponto de vista pedagógico para se conhecer como ocorre a divulgação de vídeos de neuroanatomia e para os que compartilham vídeos, pode ser interessante destacar que houve maior preferência e aceitação dos usuários por vídeos produzidos utilizando EM.

\section{REFERÊNCIAS}

1. Reidenberg JS, Laitman JT. The new face of gross anatomy. Anat Rec. 2002;269(2):81-88. DOI: 10.1002/ ar.10076.

2. Netterstrøm I, Kayser L. Learning to be a Doctor While Learning Anatomy! Anat Sci Ed. 2008;1:154-158. DOI/10.1002/ase.31.

3. Schon F, Hart P, Fernandez C. Is clinical neurology really so difficult? J Neurol Neurosurg Psychiatry. 2002;72(5):557-559. DOI:10.1136/jnnp.72.5.557.

4. Bergman EM, van der Vleuten CP, Scherpbier AJ. Why don't they know enough about anatomy? A narrative review. Med Teach. 2011;33(5):403-409. DOI: 10.3109/0142159X.2010.536276.

5. Johnson EO, Charchanti AV, Troupis TG. Modernization of an Anatomy Class: From Conceptualization 
to Implementation: A Case for Integrated Multimodal- Multidisciplinary Teaching. Anat Sci Educ. 2012;00:000-000.

6. Lowerison G, Sclater J, Schmid RF, Abrami PC. Are we using technology for learning? J. Educational Technology Systems. 2006;34(4) 401-425.

7. Jaffar AA. YouTube: An emerging tool in anatomy education. Anat Sci Educ. 2012, 5(3):158-64. DOI: 10.1002/ase.1268.

8. Judd T, Kennedy G. A five-year study of on-campus Internet use by undergraduate biomedical students. Computers \& Education. 2010;55,(4):1564-1571. DOI.org/10.1016/j.compedu.2010.06.022.

9. Reveron R. The Use of YouTube in Learning Human Anatomy by Venezuelan Medical Students. MOJ Anat \& Physiol. 2016;2(7):00075. DOI: 10.15406/mojap.2016.02.00075.

10. Azer SA. Can "YouTube" help students in learning surface anatomy? Surg Radiol Anat. 2012;34(5):465468. DOI: 10.1007/s00276-012-0935-x.

11. Raikos A, Waidyasekara P. How useful is YouTube in learning heart anatomy? Anat Sci Educ. 2014;7(1):1218. DOI:10.1002/ase.1361.

12. Melo EM, Pinheiro JT. Procedimentos legais e protocolos para utilização de cadáveres no ensino de anatomia em Pernambuco. Rev. bras. educ. med. 2010;34(2):315-323. DOI:10.1590/S010055022010000200018.

13. Silva YA, Junior EXS, Silva BN, Rodrigues GP, Souza GO, Novaes WA, Shiosaki RK, Silva TFA, Schwingel PA. Confecção de modelo neuroanatômico funcional como alternativa de ensino e aprendizagem para a disciplina de neuroanatomia. Revista Ibero-Americana de Estudos em Educação. 2017;12(3):1674-1688. DOI.org/10.21723/riaee.v12.n.3.2017.8502.

14. Shaffer K. Teaching anatomy in the digital world. N Engl J Med. 2004;351(13):1279-1281. DOI:10.1056/ NEJMp048100.

15. Estai M, Bunt $S$. Best teaching practices in anatomy education: A critical review. Ann Anat. 2016;208:151157. DOI:10.1016/j.aanat.2016.02.010. Epub 2016 Mar 17. 